\title{
SENP1 mediates TNF-induced desumoylation and cytoplasmic translocation of HIPK1 to enhance ASK1-dependent apoptosis
}

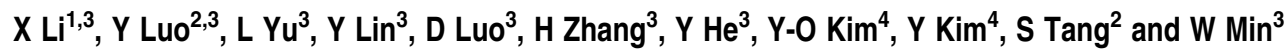

We have previously shown that tumor necrosis factor (TNF)-induced desumoylation and subsequent cytoplasmic translocation of HIPK1 are critical for ASK1-JNK activation. However, the mechanism by which TNF induces desumoylation of HIPK1 is unclear. Here, we show that SENP1, a SUMO-specific protease, specifically deconjugates SUMO from HIPK1 in vitro and in vivo. In resting endothelial cells (ECs), SENP1 is localized in the cytoplasm where it is complexed with an antioxidant protein thioredoxin. TNF induces the release of SENP1 from thioredoxin as well as nuclear translocation of SENP1. TNF-induced SENP1 nuclear translocation is specifically blocked by antioxidants such as $\mathrm{N}$-acetyl-cysteine, suggesting that TNF-induced translocation of SENP1 is ROS dependent. TNF-induced nuclear import of SENP1 kinetically correlates with HIPK1 desumoylation and cytoplasmic translocation. Furthermore, the wild-type form of SENP1 enhances, whereas the catalyticinactive mutant form or siRNA of SENP1 blocks, TNF-induced desumoylation and cytoplasmic translocation of HIPK1 as well as TNF-induced ASK1-JNK activation. More importantly, these critical functions of SENP1 in TNF signaling were further confirmed in mouse embryonic fibroblast cells derived from SENP1-knockout mice. We conclude that SENP1 mediates TNF-induced desumoylation and translocation of HIPK1, leading to an enhanced ASK1-dependent apoptosis.

Cell Death and Differentiation (2008) 15, 739-750; doi:10.1038/sj.cdd.4402303; published online 25 January 2008

The small ubiquitin-like modifier (SUMO) can be covalently attached to a large number of proteins through the formation of isopeptide bonds with specific lysine residues of target proteins. ${ }^{1,2}$ A large number of sumoylated proteins, including RanGAP1, PML, homeodomain-interacting protein kinases (HIPKs), I $\kappa$ B, p53, c-Jun, Sp3, Elk-1, p300 histone acetyltransferase, histone deacetylase (HDAC), and many nuclear receptors, have been identified. ${ }^{1-3}$ Sumoylation is a dynamic process that is mediated by activating, conjugating, and ligating enzymes and that is readily reversed by a family of SUMO-specific proteases. ${ }^{4}$ Several members of SUMOspecific proteases have been reported in the mammalian system. ${ }^{5-10}$ SENP1 is a protease that appears to deconjugate a large number of sumoylated proteins. ${ }^{5}$ Different members of these SUMO-specific proteases appear to localize in different cellular compartments where they regulate protein function by modifying the protein stability, cellular localization, and protein-protein interactions. ${ }^{5,6,8-12}$ However, it is not known how these SUMO-specific proteases are regulated.

HIPK1 is one of three closely related serine/threonine protein kinases that are primarily localized in the nucleus where it is sumoylated. ${ }^{13,14}$ The HIPKs were originally identified as nuclear protein kinases that function as co-repressors for various homeodomain-containing transcription factors. ${ }^{15}$ Recently, HIPKs have been shown to interact with other proteins involved in apoptosis and signal transduction in a cellular localization-dependent manner. In nucleus, HIPK2 phosphorylates p53 on Ser 46, resulting in the activation of p53-dependent transcription, cell growth regulation, and apoptosis initiation. ${ }^{16,17}$ HIPK2 can also promote apoptosis by downregulating the transcriptional co-repressor CtBP. ${ }^{18}$ In cytoplasm, HIPK1, HIPK2 and HIPK3 appear to transduce signals by death receptors through interaction with TRADD, FADD and apoptosis signal-regulating kinase 1 (ASK1). ${ }^{19-21}$

ASK1, a member of the mitogen-activated protein kinase kinase kinase (MAP3K) family, is an upstream activator of cJun N-terminal kinase (JNK) and p38 MAPK signaling cascades. ${ }^{22}$ ASK1 can be activated in response to diverse stresses including proinflammatory cytokine tumor necrosis factor- $\alpha$ (TNF), reactive oxygen species (ROS), death receptor Fas, disruption of microtubule structures, protein aggregation in endoplasmic reticulum (ER), and genotoxic stress from nucleus. ${ }^{23,24}$ In vitro data suggest that activation of ASK1 triggers various biological responses, such as apoptosis, inflammation, differentiation, and survival in different cell types. ${ }^{25-28}$ Studies from ASK1-deficient mice indicate that ASK1 is critical for TNF- and ROS-induced apoptosis signaling. ${ }^{29}$ The mechanism by which stress stimuli activate ASK1 is not fully understood. ASK1 activation

\footnotetext{
${ }^{1}$ The Department of Hepatobiliary Surgery, Nanfang Hospital, Southern Medical University, PR China; ${ }^{2}$ State Key Laboratory of Ophthalmology, Zhongshan Ophthalmic Center, Sun Yat-sen University, Guangzhou, PR China; ${ }^{3}$ Interdepartmental Program in Vascular Biology and Transplantation, Department of Pathology, Yale University School of Medicine, New Haven, CT, USA; ${ }^{4}$ Laboratory Research Program, National Heart, Lung, and Blood Institute, National Institutes of Health, Bethesda, MD, USA Corresponding author: W Min, Interdepartmental Program in Vascular Biology and Transplantation, Department of Pathology, Yale University School of Medicine, BCMM 454, 295 Congress Avenue, New Haven, CT 06510, USA. Tel: 203785 6047; Fax: 203737 2293; E-mail: wang.min @yale.edu

Keywords: apoptosis; ASK1; HIPK1; SENP1; TNF

Abbreviations: ASK1, apoptosis signal-regulating kinase-1; ECs, endothelial cells; HIPK1, homeodomain-interacting protein kinase 1; MEFs, mouse embryonic fibroblast cells; ROS, reactive oxygen species; SUMO, small ubiquitin-like modifier; TNF, tumor necrosis factor- $\alpha$

Received 16.1.07; revised 26.11.07; accepted 03.12.07; Edited by V De Laurenzi; published online 25.1.08
} 
appears to involve several sequential steps including association with activators such as TRAF2 ${ }^{24,30}$ and AIP1, ${ }^{31,32}$ release of cellular inhibitors such as thioredoxin and 14-3-3, ${ }^{33-35}$ and ASK1 oligomerization/autophosphorylation at Thr845. ${ }^{36}$

We have previously shown that HIPK1 is desumoylated in response to TNF leading to its cytoplasmic translocation and involvement in TNF-induced ASK-JNK signaling. ${ }^{21}$ However, the mechanism by which TNF induces HIPK1 desumoylation is not known. In the present study, we demonstrated that SENP1 mediates the TNF-induced desumoylation and the subsequent cytoplasmic translocation of HIPK1 leading to enhanced ASK1-JNK activation and endothelial cell (EC) apoptosis.

\section{Results}

SENP1 induces HIPK1 desumoylation in vitro and in vivo. It has been recently shown that SENP1 desumoylates HIPK2 in vitro and in vivo. ${ }^{37}$ To determine if SENP1 is responsible for desumoylation of HIPK1, we first performed in vitro SUMO deconjugation assays using GST-SENP1 recombinant protein containing the catalytic domain of SENP1 as we described recently. ${ }^{37}$ Consistent with previous observation, cell lysates containing Myc-HIPK1 from transient transfection of BAEC showed a sumoylated form of HIPK1 protein on the western blot (Figure 1a). The sumoylation of HIPK1 was confirmed by immunoprecipitation with anti-SUMO antibody followed by western blot with antiMyc antibody (Figure 1b; also See Figure 2b in Li et al. ${ }^{21}$ ). Addition of GST-SENP1 $(0,1$, and $2 \mu \mathrm{g})$ deconjugated SUMO-HIPK1 in a dose-dependent manner. To determine if SENP1 desumoylates HIPK1 in vivo, BAECs were transfected with Myc-HIPK1 in the presence or absence of GFP-tagged SENP1. SENP1 expression diminished SUMOHIPK1 (Figure 1b). To determine if the protease activity of SENP1 is critical for the desumoylation of HIPK1, BAECs were transfected with HIPK1 in the presence of Myc-SENP1-WT or enzymatically inactive form (SENP1-CA), which contains a mutation of $\mathrm{C} 602 \mathrm{~A}$ in the His/Asp/Cys catalytic triad. ${ }^{5} \mathrm{Co}-$ expression of SENP1-WT, but not SENP1-CA, caused disappearance of SUMO-HIPK1 (Figure 1c). Interestingly, SENP1-CA, but not SENP1-WT, showed a shifted band both in the presence or absence of HIPK1 (Figure 1c). This band was further confirmed as SUMO-SENP1 by immunoprecipitation with anti-SUMO antibody followed by western blot with anti-Myc antibody (Figure 1d). This is consistent with a recent report that SENP1 is modified by sumoylation and is self-desumoylated. ${ }^{12}$

TNF induces nuclear import of SENP1 concomitant with cytoplasmic translocation of HIPK1. It has been recently shown that SENP1 shuttles between the cytoplasm and the nucleus, and localization is cell-type dependent. ${ }^{12,37}$ To define the role of SENP1 in TNF signaling, we first determined if SENP1 localization is regulated by TNF. To this end, BAECs were transfected with GFP-SENP1 followed by treatment with TNF $(10 \mathrm{ng} / \mathrm{ml})$ for the indicated times $(0,15$, and $60 \mathrm{~min})$. Localization of GFP-SENP1 was visualized by fluorescence microscopy. In untreated cells, a majority of SENP1 is localized in the cytoplasm. However, TNF treatment for 15 min induced a translocation of SENP1 into the nucleus where it is colocalized with nuclei counterstained with DAPI (Figure 2a, top panel). Interestingly, SENP1 was detected in the cytoplasm at $60 \mathrm{~min}$, suggesting that SENP1 returned to cytoplasm from nucleus (Figure 2a, top panel). A similar pattern of SENP1 translocation was observed in other cell types such as HeLa and HUVEC (data not shown). These data suggest that SENP1 shows a reciprocal translocation of HIPK1, which is exported from nucleus to cytoplasm at $15 \mathrm{~min}$ followed by a return to nucleus at $60 \mathrm{~min}$ in response to TNF (Figure $2 \mathrm{a}$, bottom panel) (also see $\mathrm{Li}$ et al. $^{21}$ ).

TNF-induced initial translocation of HIPK1 precedes the activation of $\mathrm{JNK} / \mathrm{p} 38$ signaling. ${ }^{21}$ We reasoned that the nuclear import of SENP1 should also be an upstream event of JNK/p38 activation. To this end, we determined the effect of the JNK- or p38-specific inhibitors on TNF-induced SENP1 translocation. BAECs were transfected with GFP-SENP1, and the cells were treated with JNK inhibitor SP600125 $(20 \mu \mathrm{M})$ or p38 inhibitor SB203580 $(20 \mu \mathrm{M})$ for 30 min followed by TNF treatment $(10 \mathrm{ng} / \mathrm{ml}$ for 15 or $60 \mathrm{~min})$. Localization of GFP-SENP1 was visualized by fluorescence microscopy. SP600125 or SB203580 had no effects on the nuclear import of SENP1 induced by TNF at $15 \mathrm{~min}$ (Figure 2b). However, SP600125 (but not SB203580) specifically blocked the return of SENP1 from nucleus to cytoplasm at 60 min (Figure 2b). These data suggest that TNF-induced initial nuclear import of SENP1 precedes TNF-induced JNK activation, which is required for the subsequent return of SENP1 to cytoplasmic compartments.

Since JNK activity is required for the return of SENP1 from nucleus to cytoplasm, we reasoned that SENP1 is phosphorylated by TNF-activated JNK. To this end, BAECs were untreated or treated with TNF (10 $\mathrm{ng} / \mathrm{ml}$ for $15 \mathrm{~min})$ and cell lysates were used for an in vitro kinase assay using GSTSENP1 as a substrate in the absence or presence of a JNKspecific inhibitor SP600125 $(20 \mu \mathrm{M})$. Results showed that TNF strongly induced phosphorylation of SENP1, which was significantly blocked by the JNK-specific inhibitor (Figure 2c). As a control, TNF-induced activation of JNK (by an in vitro kinase assay using GST-C-Jun as a substrate) was also reduced by SP600125 (Figure 2d). These data suggest that TNF-activated JNK can directly phosphorylate SENP1.

TNF-induced nuclear translocation of SENP1 is ROS dependent. To determine the mechanism by which TNF induces the initial translocation of SENP1 and HIPK1, we examined the effect of $\mathrm{N}$-acetyl-cysteine (NAC), an antioxidant inhibiting TNF-induced signaling, on TNF-induced translocation of SENP1 and HIPK1. BAECs were transfected with GFP-SENP1 or GFP-HIPK1, and the cells were treated with NAC ( $1 \mathrm{mM})$ for $30 \mathrm{~min}$ followed by TNF treatment $(10 \mathrm{ng} /$ $\mathrm{ml}$ for $15 \mathrm{~min}$ ). Localization of GFP-SENP1 and GFP-HIPK1 was visualized by fluorescence microscopy. Results showed that NAC completely blocked TNF-induced nuclear import of SENP1 (Figure 3a) as well as nuclear export of HIPK1 (Figure 3b). As controls, NAC blocked TNF-induced activation 
a

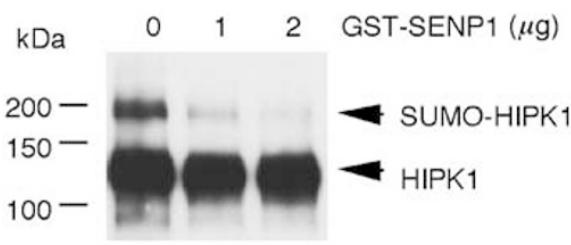

(IB: Myc)

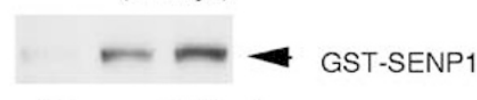

(Coomassie blue)

C

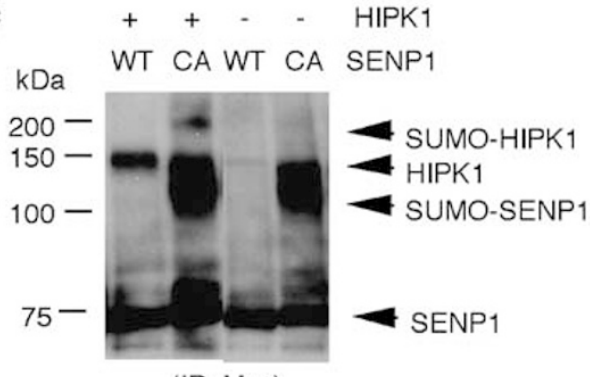

(IB: Myc)

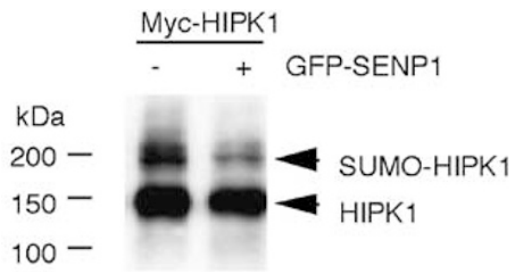

(IB: Myc)

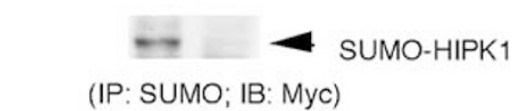

$100-\ldots$ SENP1

(IB: GFP)

d

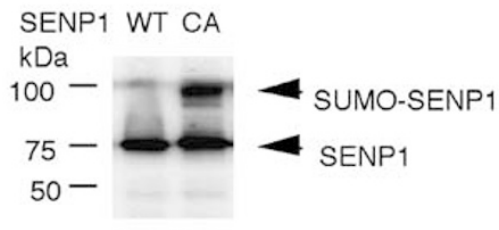

(IB: Myc)

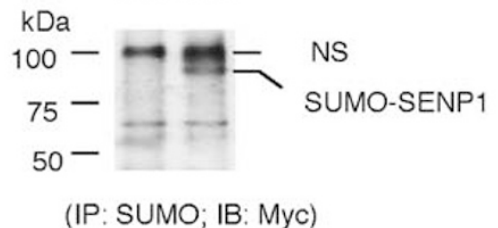

Figure 1 SENP1 deconjugates SUMO from HIPK1 in vitro and in vivo. (a) SENP1 cleaves SUMO from HIPK1 in vitro. Myc-HIPK1 (10 $\mu \mathrm{g})$ containing cell lysate was incubated with 0,1 , and $2 \mu \mathrm{g}$ of GST-SENP1 in $30 \mu \mathrm{l}$ reaction buffer containing $50 \mathrm{mM}$ Tris (pH 7.5), $2 \mathrm{mM} \mathrm{MgCl}_{2}$ and $5 \mathrm{~mm} \beta$-mercaptoethanol. Reactions were incubated at $37^{\circ} \mathrm{C}$ for $1 \mathrm{~h}$, and terminated with SDS sample buffer. Myc-HIPK1 was detected by western blot with anti-Myc antibody. (b) SENP1 cleaves SUMO from HIPK1 in vivo. BAECs were transfected with Myc-HIPK1 in the presence or absence of SENP1. HIPK1 was determined by western blot with anti-Myc antibody. SENP1 was determined by western blot with anti-GFP antibody. (c) The protease activity of SENP1 is critical for deconjugation of SUMO from HIPK1. BAECs were transfected with Myc-HIPK1 in the presence or absence of Myc-SENP1-WT or SENP-C602A. HIPK1 and SENP1 were determined by western blot with anti-Myc antibody. The single transfection of SENP1-WT and SENP1CA were used as controls. (d) SENP1 was sumoylated. The cell lysates from the single transfection of SENP1 in (c) were immunoprecipitated with anti-SUMO antibody followed by western blot with anti-Myc antibody

of JNK (Figure 3c). Hydrogen peroxide $(0.5 \mathrm{mM})$, like TNF, also induces SENP1 and HIPK1 to undergo a similar pattern of translocation (data not shown). To quantitatively analyze translocation of SENP1 and HIPK1, SENP1- or HIPK1transfected cells were subjected to cellular fractionation, and cytoplasmic and nuclear fractions were isolated according to a procedure described previously. ${ }^{38}$ The distribution of SENP1 and HIPK1 was determined by western blot with respective antibodies. ASK1 and USF2 were used as controls for cytoplasmic and nuclear fractions, respectively. Consistent with the immunofluorescence results, SENP1 protein was primarily detected in the cytoplasm of EC, and TNF induced a nuclear translocation. In contrast, HIPK1 showed a reciprocal pattern of distribution (Figure 3d). More importantly, NAC blocked TNF-induced nuclear import of SENP1 as well as nuclear export of HIPK1 (Figure 3d). These data suggest that TNF-induced nuclear translocation of SENP1 is ROS dependent.

Previously we have shown that TNF/ROS regulates ASK1 activity by dissociating redox protein thioredoxin ( $T r x)$ from ASK1 and TNF/ROS-induced dissociation of Trx from ASK1 is blocked by NAC. ${ }^{28}$ To test if SENP1 is regulated by TNF/ROS in a similar manner, we determined the SENP1-Trx complex in EC. BAECs were transfected with Myc-tagged SENP1 followed by treatment with TNF $(10 \mathrm{ng} / \mathrm{ml})$. SENP1-Trx complex was determined by co-immunoprecipitation assay using an antibody against the cytoplasmic form of Trx (Trx1). SENP1 was specifically immunoprecipitated by anti-Trx1 antibody (but not by a normal rabbit serum) in untreated cells, suggesting that SENP1 forms a complex with Trx1 in resting EC (Figure 3e). However, SENP1-Trx1 complex was disrupted in TNF-treated cells, indicating that SENP1 is dissociated from Trx1 in response to TNF (Figure 3e). These results support that TNF/ROS may induce the release of SENP1 from Trx1 in the cytoplasm, leading to a nuclear translocation of SENP1.

SENP1 induces cytoplasmic translocation of HIPK1. The data that SENP1 undergoes nuclear translocation in response to TNF prompted us to determine the role of SENP1 in TNF-induced HIPK1 desumoylation and translocation. To this end, BAECs were transfected with GFP-HIPK1 in the presence of SENP1-WT or SENP1-CA. GFP-HIPK1 was visualized under fluorescence microscope, 

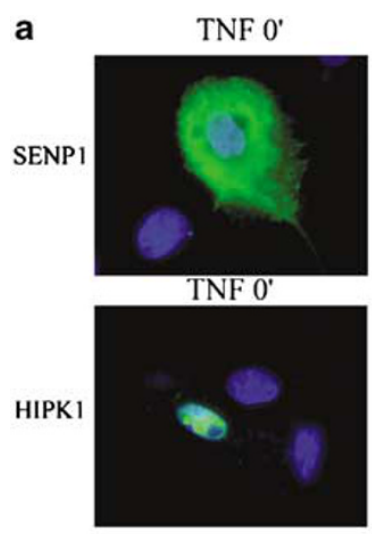

b

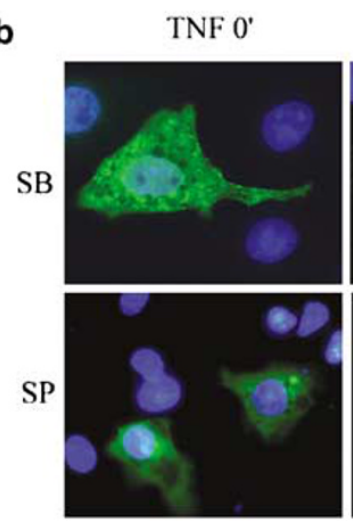

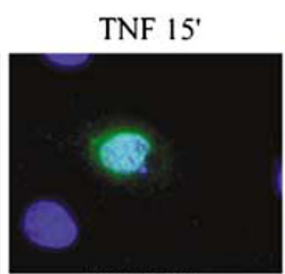

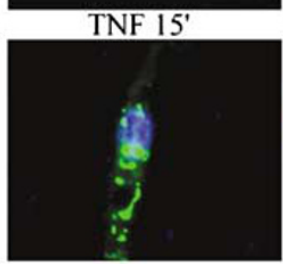

TNF $15^{\prime}$
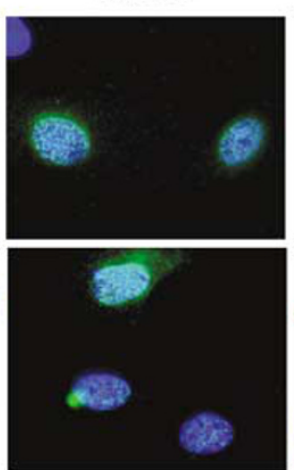

TNF $60^{\prime}$

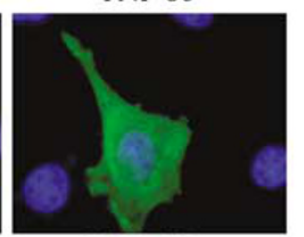

TNF $60^{\prime}$
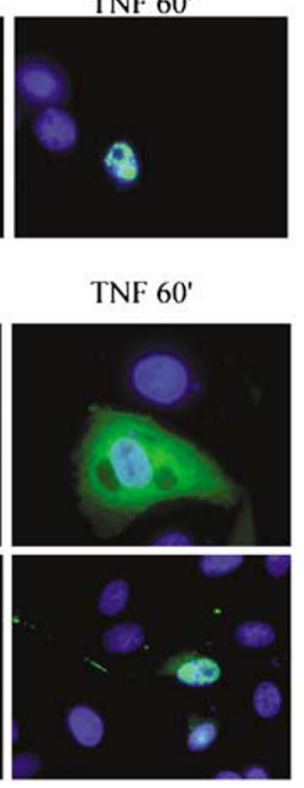

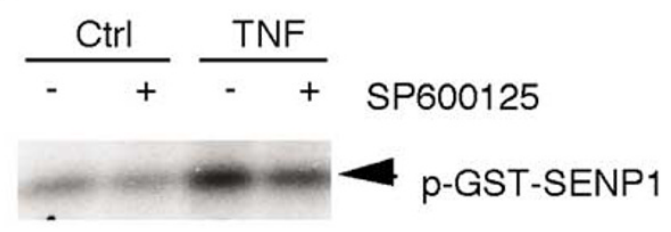

(in vitro kinase)

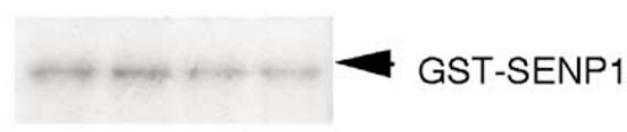

(IB: GST)

d

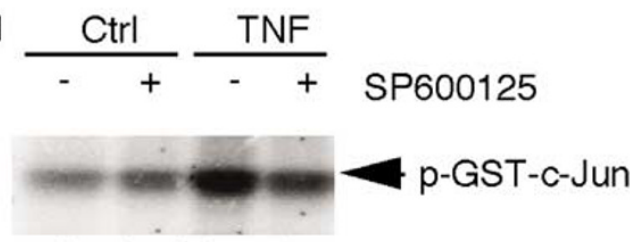

(in vitro kinase)

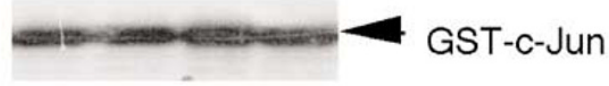

(IB: GST)

Figure 2 SENP1 is translocated from cytoplasm to nucleus in response to TNF. (a) SENP1 and HIPK1 show reciprocal translocations. BAECs were transfected with GFP. SENP1 or GFP-HIPK1 followed by treatment with TNF $(10 \mathrm{ng} / \mathrm{ml})$ for 0,15 , and $60 \mathrm{~min}$. Localization of GFP-SENP1 and GFP-HIPK1 was visualized by fluorescence microscopy. Nuclei were counterstained with DAPI. Merged images are shown on the right. (b) Effects of JNK- and p38-specific inhibitors on TNF-induced SENP1 translocation. BAECs were transfected with GFP-SENP1. Cells were treated with JNK inhibitor SP600125 (20 $\mu \mathrm{M})$ or p38 inhibitor SB203580 (20 $\mu \mathrm{M})$ for 30 min followed by TNF (10 ng/ml for 0, 15, or $60 \mathrm{~min}$ ). Localization of GFP-SENP1 was visualized by fluorescence microscopy. (c-d) TNF-activated JNK directly phosphorylates SENP1. BAECs were untreated or treated with TNF (10 ng/ml for $15 \mathrm{~min}$ ) and cell lysates were used for an in vitro kinase assay using GST-SENP1 as a substrate in the absence or presence of a JNK-specific inhibitor SP600125 (20 $\mu \mathrm{M})$. GST-c-Jun was used as a control for JNK activity (d). Total GST-SENP1 and GST-c-Jun were determined by western blot with antiGST

and SENP1 was detected by indirect immunofluorescence microscopy with anti-Myc antibody. SENP1 appeared primarily in the cytoplasm. Co-expression of SENP1-WT (Figure 4a), but not of SENP1-CA (Figure 4b), caused cytoplasmic distribution of HIPK1 prior to TNF treatment. This is consistent with that co-expression of SENP1-WT, but not SENP-CA, results in desumoylation of HIPK1 in the absence of TNF (see Figure 1b). More importantly, SENP1-CA blocked TNF-induced cytoplasmic translocation of HIPK1 (Figure 4b). In contrast, Daxx, a nuclear protein which is also localized in the nuclear body, was not translocated to cytoplasm in response to either SENP1 expression (Figure 4c) or TNF treatment, as we described previously. ${ }^{21}$

\section{A critical role of endogenous SENP1 in HIPK1} desumoylation and translocation in EC. To further confirm the physiological role of SENP1 in TNF-induced desumoylation and translocation of HIPK1, we employed siRNA to knockdown endogenous SENP1. SENP1 siRNA significantly reduced endogenous SENP1 expression compared to the control siRNA in BAEC (Figure 5a) and HeLa cells (not shown). Desumoylation of HIPK1 in response to TNF in SENP1 siRNA-expressing cells was then examined. Consistent with previous observation, TNF strongly induced desumoylation of HIPK1 (Figure 5a). Basal sumoylation of HIPK1 was not significantly altered in SENP1 siRNA-expressing cells compared to the control siRNA-expressing cells. However, SENP1 siRNA blocked TNF-induced desumoylation of HIPK1 (Figure 5a).

To determine the effect of the SENP siRNA on HIPK1 translocation, BAECs were transfected with GFP-HIPK1 in the presence of a control siRNA or the SENP1 siRNA. Cells were treated with TNF (10 ng/ml for $15 \mathrm{~min})$, and GFP-HIPK1 was visualized under fluorescence microscope. Consistently, the SENP1 siRNA, but not the control siRNA, blocked TNFinduced HIPK1 translocation (Figure 5b). Taken together, these data support that SENP1 mediates TNF-induced desumoylation and subsequent cytoplasmic translocation of HIPK1.

Critical roles of SENP1-HIPK1 in TNF-induced activation of ASK1-JNK and EC apoptosis. Given the critical role of SENP1 in HIPK1 desumoylation and translocation, we reasoned that SENP1 might play roles in TNF-induced ASK-JNK signaling and EC apoptosis. To test this hypothesis, we first examined the effects of SENP1 on 
a SENP1 TNF $0^{\circ}$
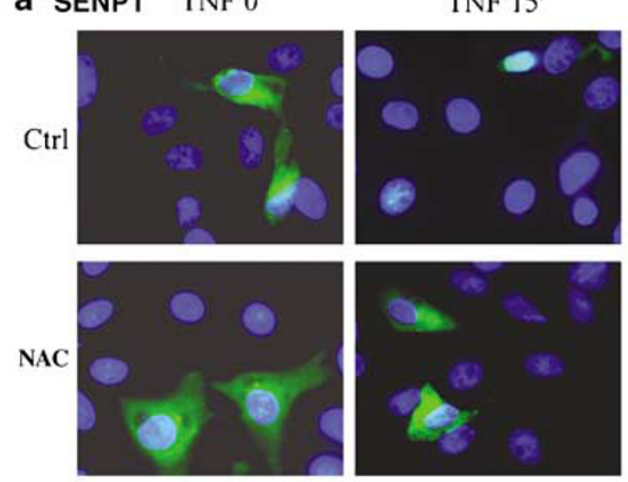

b HIPK1

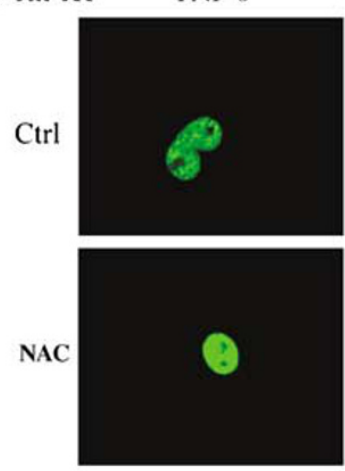

TNF $15^{\prime}$

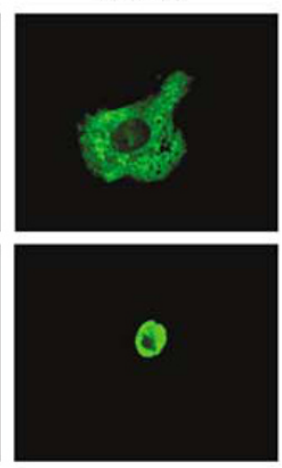

Nuclear c

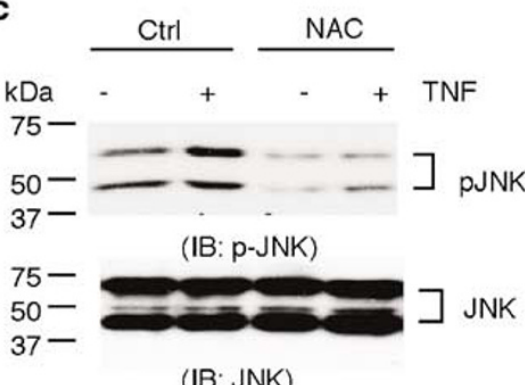

(IB: JNK)

e

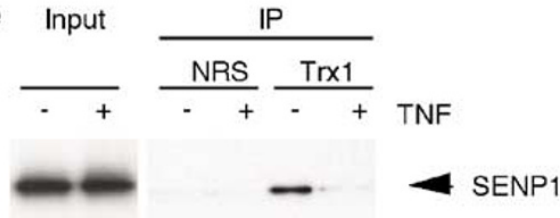

(IB: Myc)

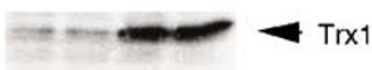

(IB: Trx1) d

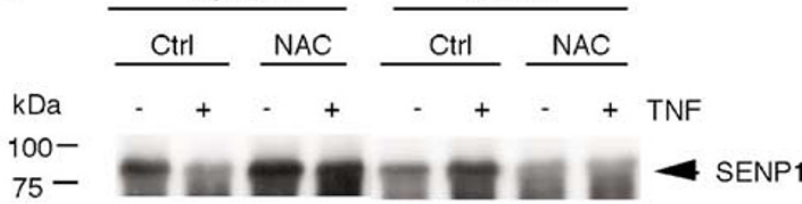

(IB: SENP1)

200$150-$

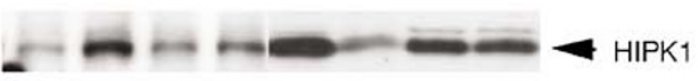

(IB: HIPK1)

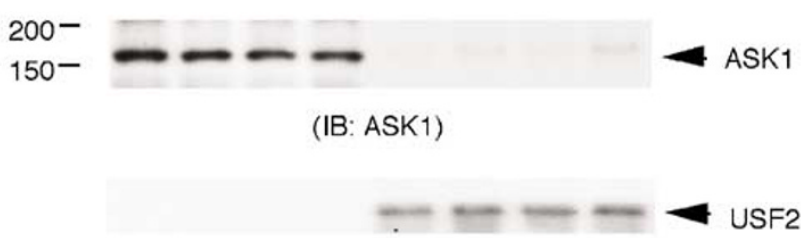

(IB: USF2)

Figure 3 Effects of antioxidants on TNF-induced initial translocations of SENP1-HIPK1. BAECs were transfected with GFP-SENP1 (a) or GFP-HIPK1 (b). Cells were treated with $\mathrm{N}$-acetyl-cysteine (NAC, $1 \mathrm{mM}$ ) for $30 \mathrm{~min}$ followed by TNF $(10 \mathrm{ng} / \mathrm{ml}$ ) for 0 or $15 \mathrm{~min}$. Localization of GFP-SENP1 and GFP-HIPK1 was visualized by fluorescence microscopy. Nuclei were counterstained with DAPI (blue) in (a). (c) Effects of Nac on TNF-induced JNK activation. BAECs were treated with Nac (1 mM) for $30 \mathrm{~min}$ followed by TNF (10 ng/ml) for 0 or $15 \mathrm{~min}$. JNK activation was determined by western blot with a phospho-specific antibody. Total JNK was determined by western blot with anti-JNK antibody. (d) Quantitative analysis of SENP1 and HIPK1 translocation by western blot. BAECs were transfected with Myc-tagged SENP1 and HIPK1 and treated as in (a, b) Cytoplasmic and nuclear fractions were isolated, and SENP1 and HIPK1 as well as endogenous ASK1 were determined by western blot with respective antibodies. ASK1 and USF2 were used as controls for cytoplasmic and nuclear fractions, respectively. (e) Regulation of SENP1-Trx complex in EC. BAECs were transfected with Myc-tagged SENP1 followed by treatment with TNF (10 ng/ml for $15 \mathrm{~min})$. SENP1-Trx complex was determined by immunoprecipitation with anti-Trx1 followed by western blot with antiMyc. A normal rabbit serum (NRS) was used as a control. Trx1 in the immunoprecipitates was determined by western blot with anti-Trx1

TNF-induced JNK reporter gene in which a c-Jun/ATF2binding site is critical for its activation. BAECs were transfected with SENP1 (WT or CA) in the presence of the JNK reporter gene. HUVECs were treated with TNF $(10 \mathrm{ng} / \mathrm{ml}$ for $6 \mathrm{~h}$ ), and the reporter gene activity was measured by luciferase assay. HIPK1-WT and HIPK1-DN were used as controls. SENP1-WT and HIPK1-WT enhanced, whereas SENP1-CA and HIPK1-DN blunted, TNF-induced activation of the JNK reporter gene (Figure 6a).

We then performed RNA interference to determine the physiological role of SENP1 in TNF-induced ASK1-JNK signaling and EC apoptosis. HUVECs were transfected with a control siRNA or SENP1 SiRNA, and cells were untreated or treated with TNF for the indicated times $(0,15$, and $60 \mathrm{~min})$.
SENP1 expression was completely knocked down by SENP1 siRNA without effects on expression of ASK1 (Figure 6b). Activation of ASK1 and JNK was determined by western blot with phospho-specific antibodies as described previously. ${ }^{39}$ Results showed that TNF-induced activation of ASK1 and JNK was significantly blunted by expression of SENP1 SiRNA (Figure 6b), highlighting the role of SENP1 in ASK1-JNK signaling pathway.

We then determined the role of SENP1 in TNF-induced cell apoptosis in SENP1 overexpression and knockdown systems. HUVECs were transfected with a control vector (VC), SENP1WT, CA, a control siRNA or SENP1 siRNA. HIPK1-WT and HIPK1-DN were used as controls. The cells were untreated or treated with TNF $(10 \mathrm{ng} / \mathrm{ml})$ plus cycloheximide $(\mathrm{CHX}, 10 \mathrm{mg} / \mathrm{ml})$ 
a
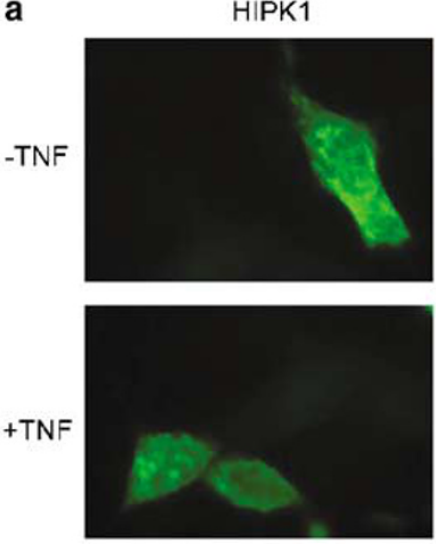

b

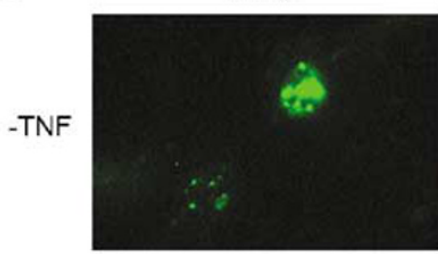

HIPK1

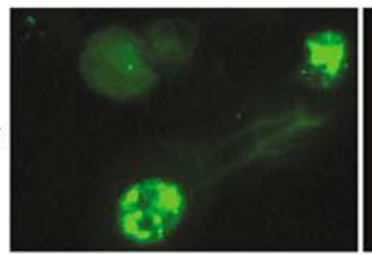

SENP1-WT
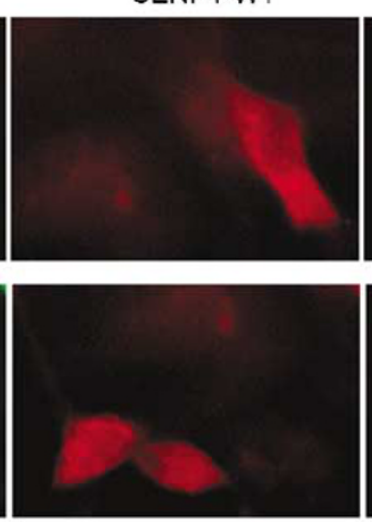

SENP1-CA
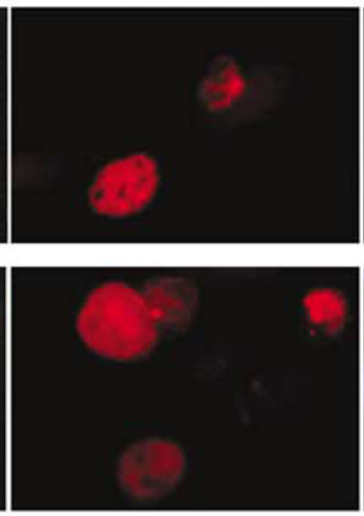

SENP1
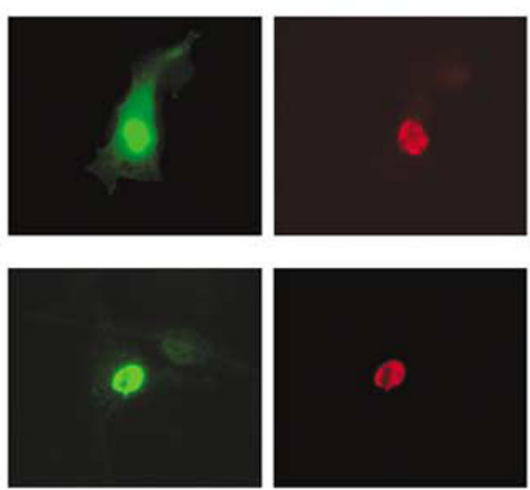

DAPI
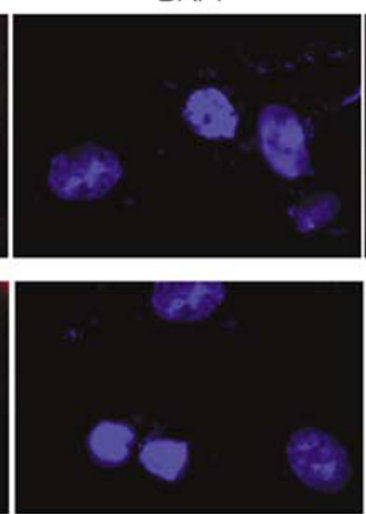

DAPI
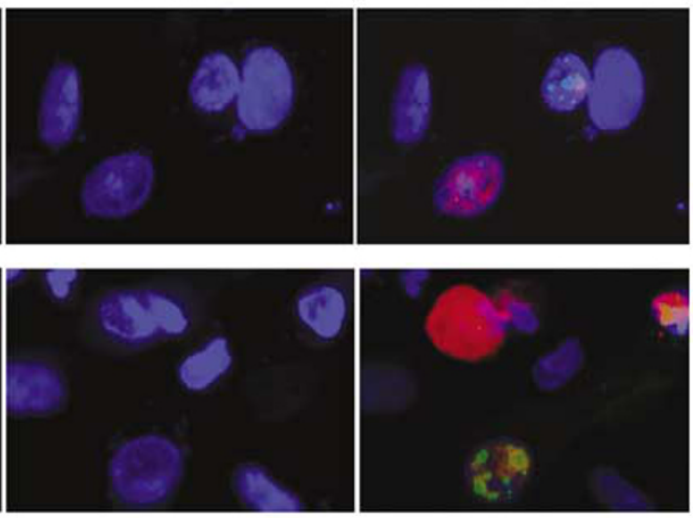

merge
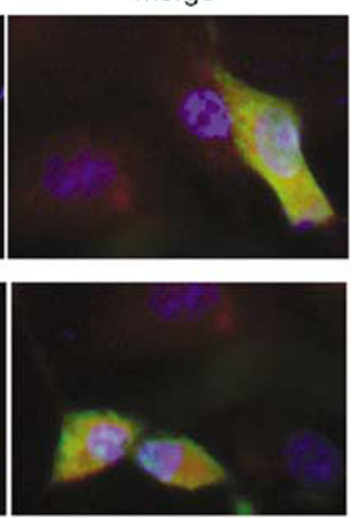

merge

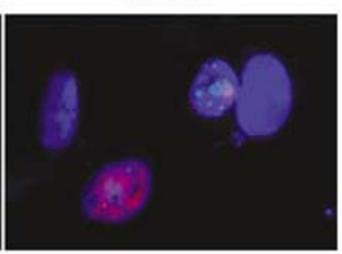

merge
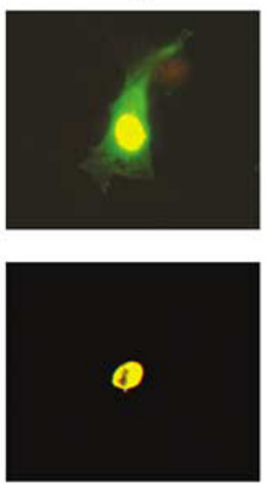

Figure 4 SENP1 induces HIPK1 cytoplasmic translocation. GFP-HIPK1 was co-transfected into BAEC with SENP1-WT (a) or SENP1-CA (b). Cells were untreated (Ctrl) or treated with TNF (10 ng/ml for $15 \mathrm{~min})$. GFP-HIPK1 was visualized by fluorescence microscopy (left). Myc-tagged SENP1 was detected by indirect immunofluorescence microscopy with anti-Myc antibody followed by Alexa 594-conjugated anti-mouse secondary antibody (center). The merged pictures are shown on the right. (c) Daxx is not translocated in response to TNF or SENP1 expression. BAECs were transfected with Daxx in the absence or presence of Myc-SENP1 followed by treatment with TNF (10 $\mathrm{ng} / \mathrm{ml})$. Daxx and SENP1 were detected by indirect immunofluorescence microscopy with anti-Myc mouse monoclonal antibody (for SENP1) and anti-Daxx rabbit polyclonal antibody followed by Alexa 488 (green)-conjugated anti-mouse and Alexa 594 (red)-conjugated anti-rabbit second antibodies, respectively. The merged pictures are shown on the right

for $6 \mathrm{~h}$. EC apoptosis was determined by DAPI staining for nuclei fragmentation. As a control, knockdown of ASK1 by siRNA of ASK1 blocked TNF (plus CHX)-induced EC apoptosis (Figure 6c). Similar to the effects of SENP1 and HIPK1 on ASK1-JNK activation, SENP1-WT and HIPK1-WT enhanced, whereas SENP1-CA, SENP1 siRNA, or HIPK1-DN significantly blocked, TNF (plus CHX)- induced EC apoptosis (Figure 6c). To further determine the critical role of ASK1 in SENP1-HIPK1 pathway, SENP1HIPK1-induced EC apoptosis was examined in ASK1-knockdown cells. Results showed that SENP1- or HIPK1-induced EC apoptosis by overexpression of SENP1 or HIPK1 was significantly reduced in ASK1-knockdown cells (Figure 6d). These data strongly support the critical roles of SENP1- 


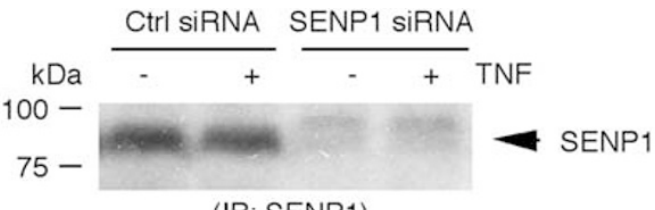

(IB: SENP1)

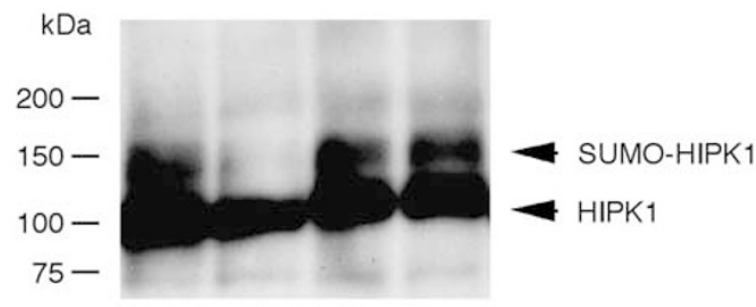

(IB: Myc)

b
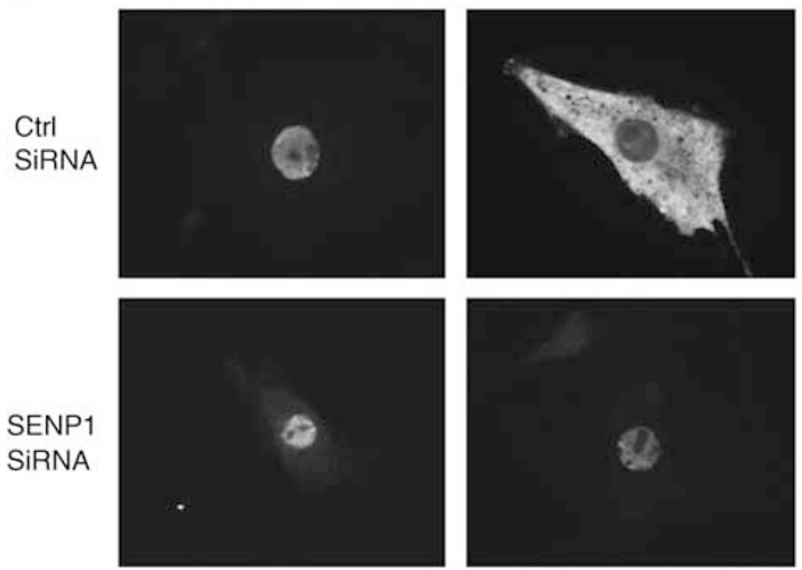

Figure 5 A critical role of SENP1 in mediating TNF-induced desumoylation and cytoplasmic translocation of HIPK1. (a) Effects of SENP1 siRNA on TNF-induced HIPK1 desumoylation. BAECs were transfected with Myc-HIPK1 in the presence of a control siRNA or SENP1 siRNA. Cells were untreated or treated with TNF (10 ng/ $\mathrm{ml}$ for $15 \mathrm{~min}$ ). Endogenous SENP1 was determined by western blot with antiSENP1 antibody. HIPK1 was determined by western blot with anti-Myc antibody. HIPK1 and SUMO-HIPK1 are indicated. (b) Effects of SENP1 siRNA on TNFinduced HIPK1 translocation. BAECs were transfected with GFP-HIPK1 in the presence of a control siRNA or SENP1 siRNA. Cells were untreated or treated with TNF $(10 \mathrm{ng} / \mathrm{ml}$ for $15 \mathrm{~min})$. Localization of GFP-HIPK1 was visualized by fluorescence microscopy

HIPK1 in TNF-induced ASK1-JNK activation and EC apoptosis.

Critical roles of SENP1 in TNF-induced desumoylation of HIPK1 and activation of ASK1-JNK in SENP1-deficient mouse embyronic fibroblast cells. To define the role of SENP1 in TNF signaling in vivo, we generated SENP1deficient mice based on a homologous recombination with a targeting vector by which the exons 5 and 6 encoding for the $\mathrm{N}$-terminal domain of SENP1 were deleted, leading to a frameshift of the downstream coding sequence. Recent reports have shown that a retrovirus insertional mutation within the mouse SENP1 gene caused downregulation of SENP1 expression and embryonic lethality at E12.5E14.5. ${ }^{40,41}$ Although mice with genetic deficiency in SENP1 (SENP1-KO) in our system were prenatal lethal (these findings will be described elsewhere), we were able to isolate mouse embryonic fibroblast cells (MEFs) from wildtype or SENP1-KO embryos at E13. SENP1 expression in MEFs was determined by western blot with anti-SENP1 antibody and results confirmed a defect of SENP1 in SENP1KO MEF (Figure 7a).

To determine the effects of SENP1 deficiency on sumoylation of HIPK1, MEFs were treated with TNF $(10 \mathrm{ng} / \mathrm{ml})$ for indicated times $(0,15$, and $60 \mathrm{~min})$. TNF-induced desumoylation of HIPK1 in WT and SENP1-KO MEFs was determined by western blot with anti-HIPK1. Similar to SENP1 knockdown by siRNA, the basal sumoylation of HIPK1 was not significantly altered in SENP1-KO MEF compared to that in WT MEF. TNF strongly induced desumoylation of HIPK1 in WT MEF. However, TNF-induced desumoylation of HIPK1 was completely blocked in SENP1-KO MEF (Figure 7b). To determine the effect of SENP1 deficiency on TNF-induced HIPK1 translocation, WT and SENP1-KO MEFs were transiently transfected with GFP-HIPK1 followed by TNF treatment for 15 and $60 \mathrm{~min}$. GFP1-HIPK1 was determined by fluorescence microscopy. Similar to SENP1 knockdown by siRNA, SENP1 deficiency also blocked TNF-induced nuclear export of HIPK1 (not shown). These data further support that SENP1 mediates TNF-induced desumoylation and cytoplasmic translocation of HIPK1.

Next, we determined the effect of SENP1 deficiency on TNF-induced ASK1-JNK signaling and cell death. WT and SENP1-KO MEFs were treated with TNF for the indicated times $(0,15$, and $60 \mathrm{~min})$. Activation of ASK1 and JNK was determined by western blot with phospho-specific antibodies. Results showed that TNF-induced activation of ASK1 and JNK was significantly blunted in SENP1-KO MEF compared to WT MEF (Figure 7c), confirming the role of SENP1 in ASK1-JNK signaling pathway. To determine the role of SENP1 in TNF-induced cell apoptosis, WT and SENP1-KO MEFs were treated with TNF $(10 \mathrm{ng} / \mathrm{ml})$ plus cycloheximide $(\mathrm{CHX}, 10 \mathrm{mg} / \mathrm{ml})$ for $6 \mathrm{~h}$. Apoptosis and necrosis of the MEFs were determined by $\mathrm{PI}$ exclusion and annexin $\mathrm{V}$ staining followed by FACS analysis. TNF (plus CHX) significantly induced apoptosis but not necrosis. Moreover, TNF $(+\mathrm{CHX})$ induced apoptosis was significantly blunted in SENP1-KO MEFs compared to WT MEFs (Figure 7d with quantification apoptosis in Figure 7e and necrosis in Figure 7f). These data support the critical roles of SENP1-HIPK1 in TNF-induced ASK1-JNK activation and EC apoptosis.

\section{Discussion}

In view of our data, we propose a following model for the role of SENP1-HIPK1 in ASK1-JNK signaling (Figure 8). In resting EC, SENP1 is localized in the cytoplasm where it is complexed with Trx1. HIPK1 is sumoylated and localized in the nucleus. In response to stress stimuli including TNF and ROS, SENP1 is dissociated from Trx1 and translocated into nucleus where it desumoylates HIPK1. Desumoylated HIPK1 is exported to cytoplasm where it binds to the AIP1-ASK1 signaling complex leading to activation of ASK1-JNK signaling and EC apoptosis. ${ }^{21}$

The most important finding of our present study is that we have defined the mechanism by which TNF induces SENP1 

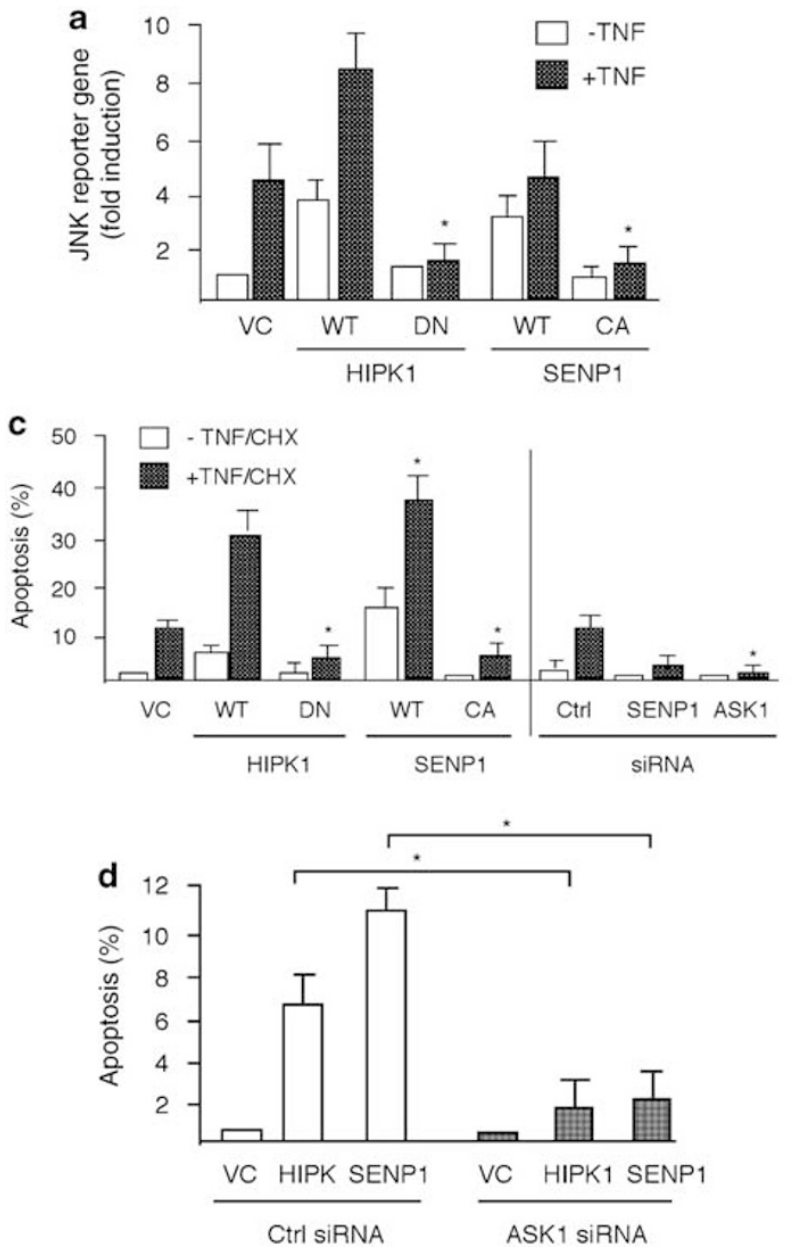

b

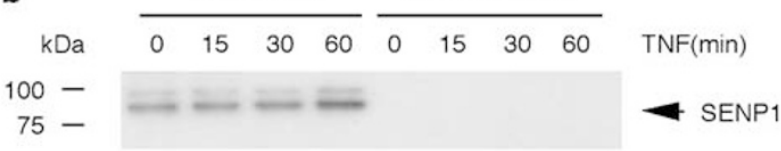

(IB: SENP1)

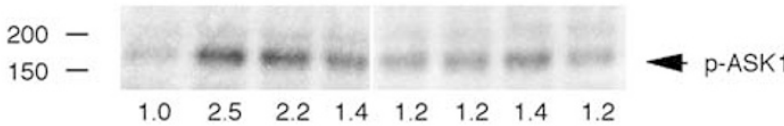

(IB: p-T845)

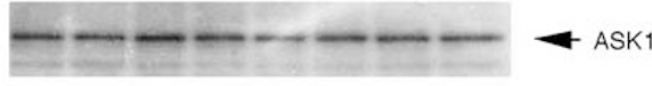

(IB: ASK1)
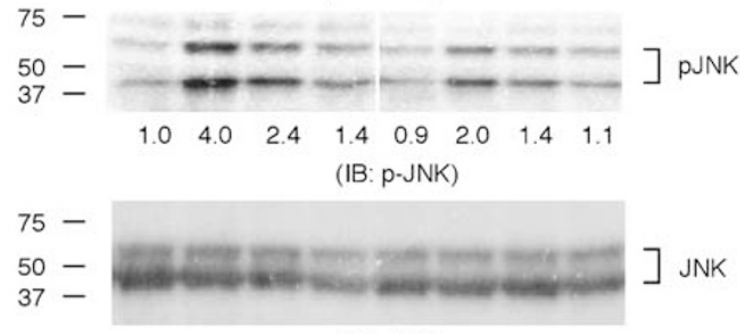

(IB: JNK)

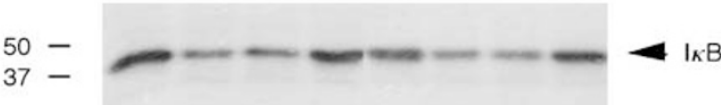

$(\mathrm{IB}: 1 \kappa \mathrm{B} \alpha)$

Figure 6 Critical roles of SENP1-HIPK1 in TNF-induced activation of ASK1-JNK signaling. (a) SENP1-WT increased while SENP1-CA blocked TNF-induced activation of JNK-dependent reporter gene. HUVECs were transfected with SENP1-WT or -CA in the presence of a JNK reporter gene. A renilla construct was co-transfected as an internal control. HIPK1-WT and -DN were used as controls. Cells were treated with TNF for $6 \mathrm{~h}$, and both luciferase and renilla units were measured. Relative luciferase activities are presented from mean of duplicate samples by taking untreated vector control as 1 . Similar results were obtained from two additional experiments. Data are presented as mean of duplicates from two independent experiments. (b) SENP1 siRNA blocks TNF-induced activation of ASK1-JNK. HUVECs were transfected with a control or SENP1 siRNA. At $72 \mathrm{~h}$ post-transfection, cells were treated with $\operatorname{TNF}(10 \mathrm{ng} / \mathrm{ml})$ for various times $(0,15,30$, or $60 \mathrm{~min})$. Total protein of SENP1 and ASK1 was determined by western blot with respective antibodies. Activation of ASK1 and JNK activity was determined by western blot with phospho-specific antibodies. Relative ratios of pASK1/ASK1 and p-JNK/JNK are shown, with untreated VC as 1.0. (c, d) Critical roles of SENP1 and ASK1 in TNF (plus cycloheximide; CHX)-induced EC apoptosis. HUVECs were transfected with SENP1-WT, -CA, a control siRNA, SENP1 siRNA or ASK1 siRNA. HIPK1-WT and -DN were used as controls. At $72 \mathrm{~h}$ post-transfection, cells were treated with TNF (10 ng/ml) plus $\mathrm{CHX}(10 \mu \mathrm{g} / \mathrm{ml})$ for $6 \mathrm{~h}$. EC apoptosis was determined by DAPI staining for nuclei fragmentation. (d) BAECs were transfected with HIPK1-WT and SENP1-WT, in the presence of a control siRNA or ASK1 siRNA. EC apoptosis was determined by DAPI staining for nuclei fragmentation. The apoptosis rate is shown. Data are presented as mean of duplicates from two independent experiments. *Statistically significant compared to VC, $P<0.05$

translocation and identified a unique SENP1-HIPK1 signaling pathway-mediated TNF-induced ASK1-JNK activation. Different from classic TNF-induced 'membrane to cytoplasm' mediated by TNFR1-TRADD-TRAF2, several sequential steps are involved in the SENP1-HIPK1 signaling pathway including (1) dissociation of SENP1 from Trx1; (2) SENP1 nuclear import; (3) desumoylation of HIPK1 and nuclear export; and (4) association of HIPK1 with AIP1-ASK1 complex leading to ASK1-JNK activation. It is not known whether the TNFR1-TRADD-TRAF2 pathway cross talks to the SENP-HIPK1 pathway. A dominant-negative TRAF2 does not block SENP1-HIPK1-transduced ASK1 activation, suggesting that these two pathways may converge on AIP1 which associates with both TRAF2 ${ }^{32}$ and HIPK $1 .{ }^{21}$ It has been previously reported that in response to Fas engagement,
Daxx is translocated from nucleus to cytoplasm where it associates with and activates ASK $1 .{ }^{24}$ Moreover, Daxxinduced ASK1 activation is mediated by interaction of Daxx to the N-terminal domain of ASK1, and the kinase activity of ASK1 is not critical for Daxx-ASK1-mediated apoptosis. ${ }^{42}$ We did not observe Daxx cytoplasmic translocation in response to TNF. ${ }^{21}$ Furthermore, SENP1 does not regulate Daxx cytoplasmic translocation, suggesting that a specific role of SENP1 in TNF-induced ASK1-JNK signaling pathway.

Translocation of SENP1 from cytoplasm to nucleus appears to be an early step prior to the stress stimuli-induced desumoylation and subsequent nuclear export of HIPK1 and ASK1-JNK signaling. This is supported by the data that the wild-type SENP1 induces the desumoylation and nuclear export of HIPK1, whereas an enzymatic inactive mutant of 


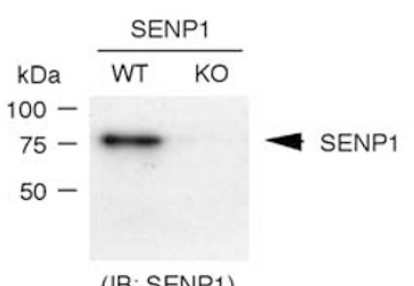

(IB: SENP1)

C

$200-$
$150-$

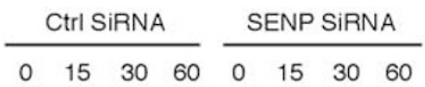

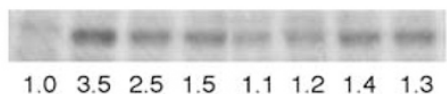

4 p-ASK

(IB: p-T845)

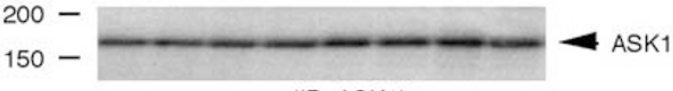

(IB: ASK1)

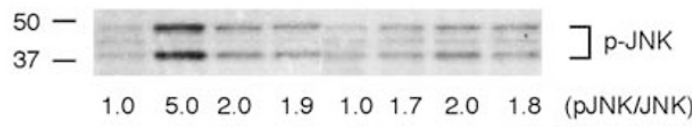

(IB: pJNK)

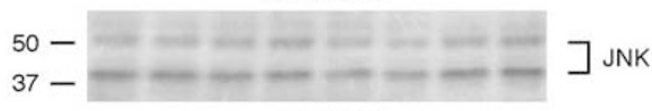

(IB: JNK)

e

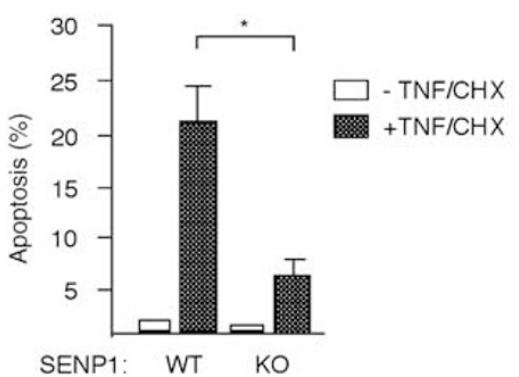

b

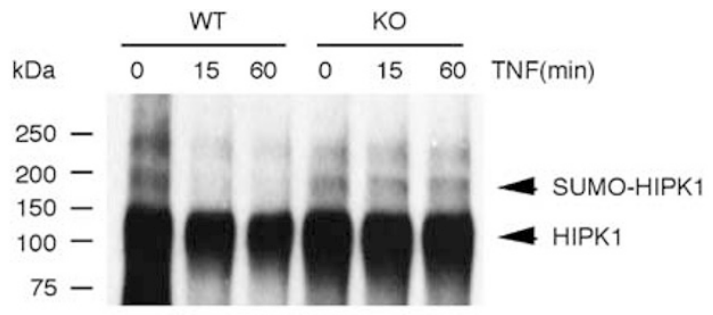

(IB: HIPK1)

d
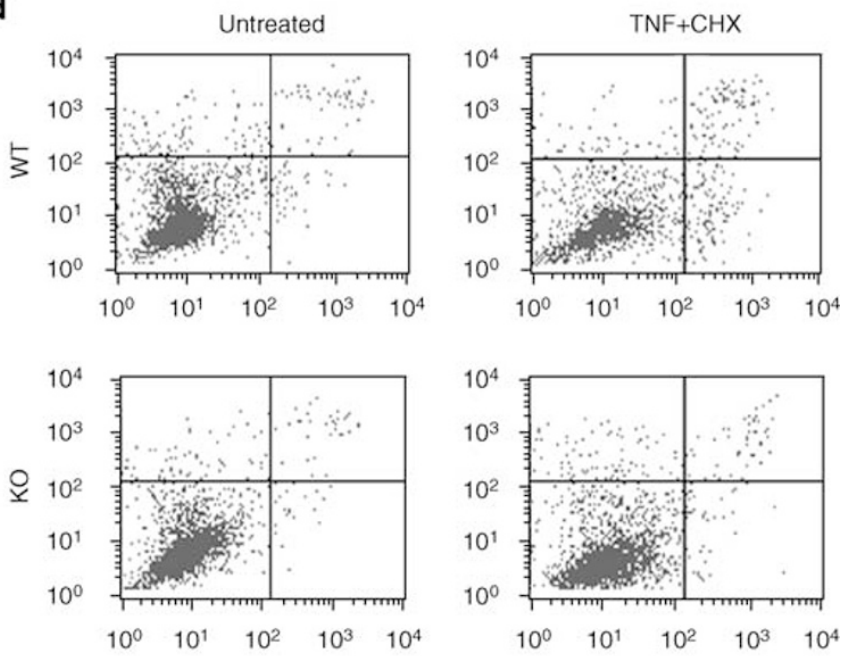

f

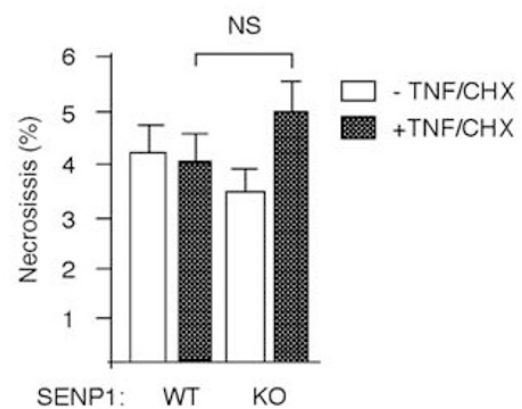

Figure 7 Critical roles of SENP1 in TNF-induced HIPK1 desumoylation and activation of ASK1-JNK apoptotic signaling. (a) Mouse embryonic fibroblast cells (MEFs) were isolated from wild-type or SENP1-KO embryos at E13.3 SENP1 expression in MEF were determined by western blot with anti-SENP1 antibody. (b) MEFs were treated with $\operatorname{TNF}(10 \mathrm{ng} / \mathrm{ml})$ for indicated times $(0,15$, and $60 \mathrm{~min})$. TNF-induced desumoylation of HIPK1 was determined by western blot with anti-HIPK1. (c) WT and SENP1-KO MEFs were treated with TNF for the indicated times $(0,15,30$, and $60 \mathrm{~min})$. Activation of ASK1 and JNK was determined by western blot with phospho-specific antibodies. (d-f) WT and SENP1-KO MEFs were untreated or treated with TNF $(10 \mathrm{ng} / \mathrm{ml})$ plus cycloheximide (CHX, $10 \mathrm{mg} / \mathrm{ml})$ for $6 \mathrm{~h}$. MEF apoptosis and necrosis were determined by PI-annexin V staining followed by FACS analysis. Representative FACS is shown in (d) and the percentage of apoptotic cells (both upper and lower right quadruplets in FACS) and necrosis (upper left) is quantified in (e, f), respectively. Data are presented as mean of duplicates from two independent experiments. * Statistically significant between WT and SENP1-KO MEFs, $P<0.05$; NS, non-significance

SENP1, SENP1 siRNA or SENP1 deficiency inhibits TNF/ ROS-induced desumoylation, nuclear export of HIPK1, and activation of ASK1-JNK. These data have established a critical role of SENP1 in mediating TNF-induced desumoylation and cytoplasmic translocation of HIPK1.

We have defined a potential mechanism by which TNF induces nuclear translocation of SENP1. SENP1 forms a preexisting complex with a cytoplasmic redox protein Trx 1 , and TNF induces the release of SENP1 from Trx1, suggesting that dissociation of SENP1 from Trx1 may be a critical step regulating SENP1 nuclear translocation. This is supported by the results that an antioxidant NAC completely blocks TNFinduced nuclear translocation of SENP1 and subsequent HIPK1 cytoplasmic translocation. In contrast, inhibition of JNK (by inhibitor SP600125) or p38 (by inhibitor SB203580) had no effects on the nuclear translocation of SENP1. Together with the functional studies, it is likely that TNF-induced nuclear translocation of SENP1 precedes TNF-induced ASK1-JNK/ p38 activation. It has been shown that SENP1 contains a single non-consensus nuclear localization signal (NLS) within the $\mathrm{N}$ terminus of the protein. ${ }^{12}$ It is conceivable that the Trx1 binding may block the NLS of SENP1 or alter SENP1 


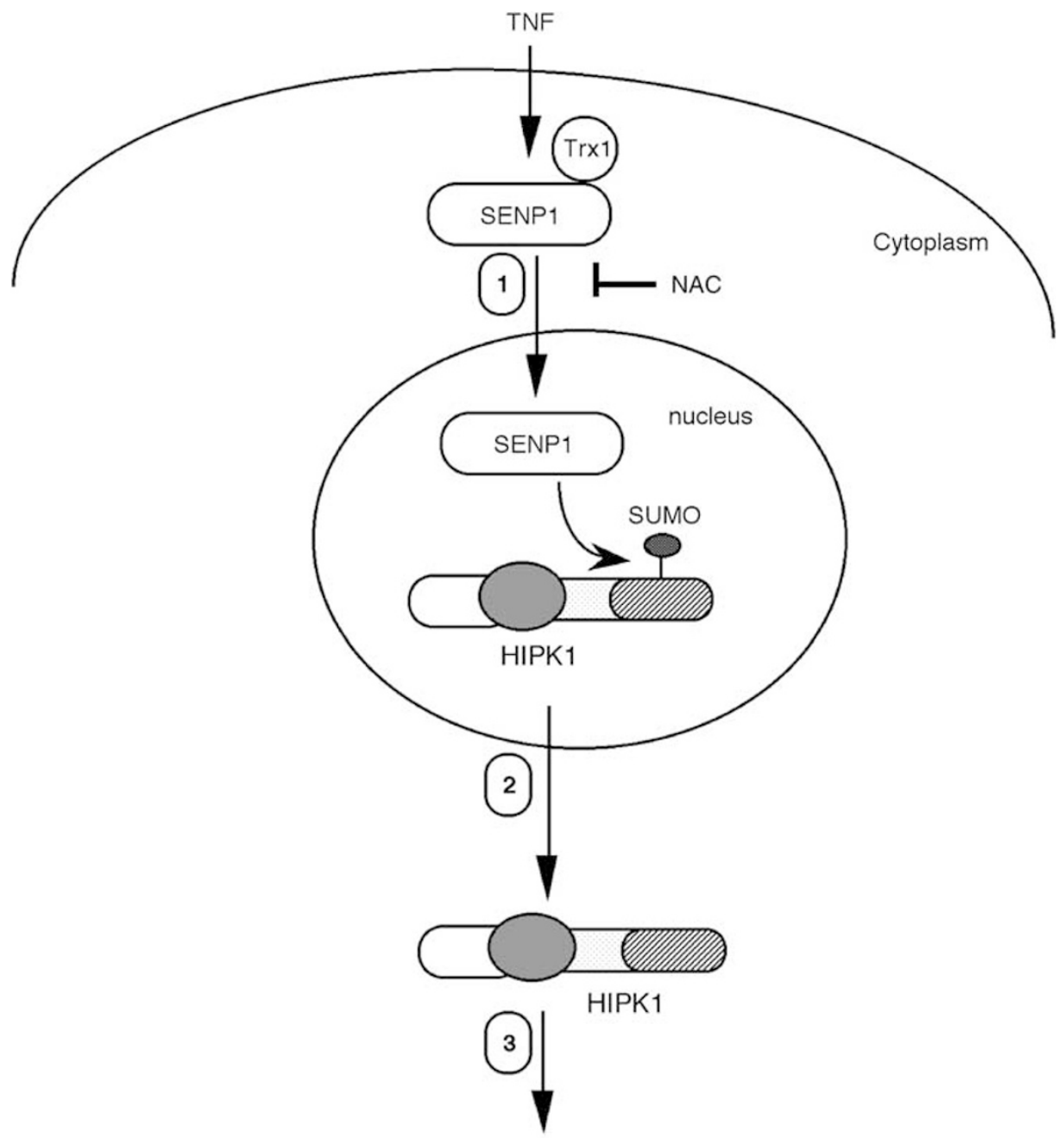

ASK1-JNK activation Apoptosis

Figure 8 A model for regulation of ASK1-JNK/p38 signaling by SENP1-HIPK1. SENP1 is localized in the cytoplasm, while HIPK1 in the nucleus in resting EC. TNF induces translocation of SENP1 (from cytoplasm to nucleus) in a ROS-dependent manner. Nuclear-located SENP1 induces desumoylation of HIPK1 and subsequent cytoplasmic translocation of HIPK1. TNF also induces a translocation of AIP1 (from membrane to cytoplasm) to form HIPK1-AIP1-ASK1 ternary complex leading to dissociation of thioredoxin and 14-3-3 from ASK1 and subsequent JNK/p38 activation ${ }^{21}$

conformation to prevent an interaction of SENP1 with the nuclear import machinery. These possibilities are currently under investigation. Interestingly, we show that SP600125 (but not SB203580) specifically blocked the return of SENP1 from nucleus to cytoplasm (this study) and subsequent HIPK1 from cytoplasm to nucleus at $60 \mathrm{~min} .{ }^{21}$ Furthermore, JNK can directly phosphorylate SENP1. Thus, JNK acts as a feedback mechanism to regulate subsequent nuclear export of SENP1. SENP1 nuclear distribution could be influenced by expression and localization of SUMO-conjugated target proteins within the cells. ${ }^{12}$ Furthermore, SENP1 contains a nuclear export sequence (NES). It is possible that SENP1 phosphorylation by JNK may induce an exposure of the NES or disruption of SENP1 interactions with other proteins, leading to return of SENP1 to cytoplasm.

In conclusion, our data show that SENP1 desumoylates HIPK1 and induces HIPK1 cytoplasm translocation, leading to enhanced ASK1-JNK activation, and suggest that SENP1HIPK1 transduces a novel pathway in TNF-induced ASK1 activation.

\section{Materials and Methods}

Plasmid construction. Expression plasmids for Daxx were generously provided by Dr. Jacques Landry (Universite Laval, Quebec, Canada) and SUMO from Dr. Ronald T Hay (University of St Andrews, Scotland). The expression plasmids for GFP- and Myc-tagged SENP1 and HIPK1 were generated by inserting human SENP1 or mouse HIPK1 cDNA into pEGFP-C2 and pCS3 + MTBX, respectively, as described previously for HIPK2. ${ }^{13,15}$ The mutant SENP1 (C602A) was constructed by site-directed mutagenesis using Quickchange ${ }^{\mathrm{TM}}$ site-directed mutagenesis kit (Stratagene) according to the protocol of the manufacturer.

Antibodies. A rabbit polyclonal anti-phospho-specific antibody against phopsho-ASK1 (pThr845) and phospho-p38 from Cell Signaling and phosphoJNK from Biosource. We obtained anti-ASK1 (H300), anti-p38, anti-Myc, and antiSUMO from Santa Cruz Biotechnology, Santa Cruz, CA, USA. Anti-HA was from 
Roche and anti-Flag was from Sigma. Rabbit polyclonal antibodies against SENP1 and HIPK1 were generated in our laboratories.

Cells, cytokines, and inhibitors. Human umbilical vein ECs (HUVECs) and bovine aortic ECs (BAECs) were purchased from Clonetics Corp. (San Diego CA, USA). HUVECs were cultured in modified M199 culture medium, containing $20 \% \mathrm{v} / \mathrm{v}$ heat-inactivated bovine fetal calf serum, $100 \mathrm{mg} / \mathrm{ml}$ heparin sodium salt, $30 \mathrm{mg} / \mathrm{ml} \mathrm{EC}$ growth supplement, $2 \mathrm{mM} \mathrm{L-glutamine,} 60 \mathrm{U} / \mathrm{ml}$ penicillin, and $0.5 \mathrm{mg} /$ $\mathrm{ml}$ streptomycin at $37^{\circ} \mathrm{C}$, in $5 \% \mathrm{CO}_{2}$ on gelatin-coated tissue culture plastic. Cells were used at passages $2-4$. Human recombinant TNF was from R\&D Systems Inc. (Minneapolis, MN, USA) and used at $10 \mathrm{ng} / \mathrm{ml}$. Chemical inhibitors were purchased from Calbiochem.

Transfection, reporter gene assay, and in vitro kinase assay. Transfection of HUVEC was performed by DEAE-Dextran method and BAECs were transfected by Lipofactamine 2000 (Gibco) as described. ${ }^{27,31,32}$ Luciferase activity followed by renilla activity was measured twice in duplicate using a Berthold luminometer. All data were normalized as relative luciferase light units/ renilla unit. In vitro kinase assay using GST-C-Jun and GST-SENP1 was performed as described previously. ${ }^{27,31,32}$

Immunoprecipitation and immunoblotting. HUVECs or BAECs after various treatments were washed twice with cold PBS and lysed in $1.5 \mathrm{ml}$ of cold lysis buffer $(50 \mathrm{mM}$ Tris- $\mathrm{HCl}, \mathrm{pH} 7.6,150 \mathrm{mM} \mathrm{NaCl}, 0.1 \%$ Triton X-100, $0.75 \%$ Bri] 96, $1 \mathrm{mM}$ sodium orthovanadate, $1 \mathrm{mM}$ sodium fluoride, $1 \mathrm{mM}$ sodium pyrophosphate, $10 \mathrm{mg} / \mathrm{ml}$ aprotinin, $10 \mathrm{mg} / \mathrm{ml}$ leupeptin, $2 \mathrm{mM}$ PMSF and $1 \mathrm{mM}$ EDTA) for $20 \mathrm{~min}$ on ice. For immunoprecipitation to analyze protein interaction in vivo, supernatants of cell lysates were precleared by incubating with normal rabbit serum plus GammaBind plus Sepharose beads on rotator at $4^{\circ} \mathrm{C}$ overnight. The lysates were then incubated with the first protein-specific antiserum (e.g. antiSUMO-1 from Santa Cruz Biotechnology) for $2 \mathrm{~h}$ with $50 \mathrm{ml}$ of GammaBind plus Sepharose. Immune complexes were collected after each immunoprecipitation by centrifugation at $13000 \mathrm{~g}$ for $10 \mathrm{~min}$ followed by $3-5$ washes with lysis buffer. The immune complexes were subjected to SDS-PAGE followed by immunoblot with the second protein (e.g. HIPK1)-specific antibody. The chemiluminescence was detected using an ECL kit according to the instructions of the manufacturer (Amersham Life Science, Arlington Heights, IL, USA). For detection of GFP- and Myc-tagged proteins (SENP1 and HIPK1), anti-GFP antibody (Santa Cruz Biotechnology) and anti-Myc (Roche Diagnostics) were used for immunoblotting, respectively.

Indirect immunofluorescence confocal microscopy. Fixation permeabilization, and staining of cultured EC were performed as described previously. ${ }^{32}$ Alexa Fluor 488 (green) or 594 (red) conjugated-secondary antibodies (Molecular Probes, Eugene, OR, USA) were used. Confocal immunofluorescence microscopy was performed using an Olympus confocal microscope under a $\times 63$ optical objective at a single optical section, and acquired images were transferred to Photoshop 6.0 to generate the final figures.

RNAi for SENP1 and ASK1. In view of the established characteristics of siRNA-targeting constructs, ${ }^{43}$ we designed a pair of siRNA oligonucleotides for human SENP1: $5^{\prime}$ GGACCAGCUUUCGCUUUCUdTdT3'. The corresponding scramble siRNA oligonucelotide: 5'GGACCAGCAUACGCUUUCUdTdT3' with two nucleotide mutations (underlined) was synthesized from Ambion (Austin, TX USA). siRNA for human ASK1 and control siRNA were purchased from Santa Cruz Biotechnology. siRNAs $(20 \mu \mathrm{M})$ were transfected into cells by Oligofectamine following protocols provided by the manufacturer (Invitrogen, Carlsbad, CA, USA). At $72 \mathrm{~h}$ post-transfection, cells were harvested for protein analyses as described.

Quantitation of apoptotic cell. The propidium iodide (PI) exclusion method for loss of integrity of cell membranes was used to assess viability as we described previously. ${ }^{44}$ In brief, cells were suspended in PBS containing $25 \mu \mathrm{g} / \mathrm{ml} \mathrm{PI}$ for $5 \mathrm{~min}$ at $37^{\circ} \mathrm{C}$ and then subjected to analytic flow cytometry on a FACSort (BD Biosciences) immediately after labeling. A light scatter gate was set up to eliminate cell debris from the analysis. The PI fluorescence signal was recorded on the FL3 channel and analyzed by using CellQuest software. Phosphatidylserine translocation, which precedes loss of PI exclusion in apoptotic cell death, was assessed by an annexin V-FITC staining kit (Roche) following the manufacturer's protocol. For nuclear morphology, cells were stained with DAPI and apoptotic cells (nuclei condensation) were visualized under UV microscope.

Targeted inactivation of SENP1 gene by homologous recombination and isolation of MEFs. The SENP1-targeting arms were isolated from BAC clone identified by screening a Research Genetics (Invitrogen) BAC library using SENP1 CDNA as a probe. The targeting vector was constructed in pEASYflox backbone to contain a loxP site inserted upstream of SENP1 exon 5, and a neomycin cassette (Neo) flanked by two loxP sites downstream of exon 6 using standard molecular procedures. The linearized targeting construct was electroporated into 129/C57B/6 ES cells, and the targeted clones were selected with G418 and gancyclovir. Resistant clones were screened for homologous recombination by PCR and confirmed by Southern blot analysis. Two independent SENP1 ${ }^{\text {lox }}$ ES cell clones were injected into C57BL/6 blastocysts. Chimeras were further bred with $\mathrm{C} 57 \mathrm{BL} / 6$ female for germline transmission. SENP1 ${ }^{10 x / l o x}$ mice were mated with $\beta$-actin-Cre mice to mediate a recombination in vivo, resulting in a complete deletion of SENP1 exons 5 and 6 and a frameshift of the downstream catalytic domain. MEFs were established from embryos at embryonic day 13. Briefly, the matings of SENP1-KO mice were timed and the embryos generated were dissected at E13. Yolk sacs were isolated and genotyped with the use of PCR; each individual embryo was homogenized and treated with trypsin for $30 \mathrm{~min}$. After washing with Dulbecco's modified Eagle's medium (DMEM, $1 \mathrm{ml}$ ) containing $15 \%$ fetal bovine serum (FBS), the embryonic fibroblast cells were cultured in six-well plates that had been coated with $0.1 \%$ gelatin in DMEM with $15 \%$ FBS. Genotypes of the established MEFs were further confirmed by Southern blot and PCR analysis. MEFs used for all experiments were primary cells between passages 3 and 7 .

Acknowledgements. This study was supported by $\mathrm{NIH}$ grants R01 HL65978-5, R01 HL077357-1, and P01HL070295-6 and an Established Investigator Award from the American Heart Association (0440172N) to WM.

1. Muller S, Hoege C, Pyrowolakis G, Jentsch S. SUMO, ubiquitin's mysterious cousin. Nat Rev Mol Cell Biol 2001; 2: 202-210.

2. Pickart CM. Mechanisms underlying ubiquitination. Annu Rev Biochem 2001; 70: 503-533.

3. Cheng J, Wang D, Wang Z, Yeh ET. SENP1 enhances androgen receptor-dependent transcription through desumoylation of histone deacetylase 1. Mol Cell Biol 2004; 24: 6021-6028.

4. Li SJ, Hochstrasser M. A new protease required for cell-cycle progression in yeast. Nature 1999; 398: 246-251.

5. Gong L, Millas S, Maul GG, Yeh ET. Differential regulation of sentrinized proteins by a novel sentrin-specific protease. J Biol Chem 2000; 275: 3355-3359.

6. Suzuki T, Ichiyama A, Saitoh $\mathrm{H}$, Kawakami T, Omata $\mathrm{M}$, Chung $\mathrm{CH}$ et al. A new 30-kDa ubiquitin-related SUMO-1 hydrolase from bovine brain. J Biol Chem 1999; 274: 31131-31134

7. Kim KI, Baek SH, Jeon YJ, Nishimori S, Suzuki T, Uchida S et al. A new SUMO-1-specific protease, SUSP1, that is highly expressed in reproductive organs. J Biol Chem 2000; 275: 14102-14106.

8. Kadoya T, Yamamoto $H$, Suzuki T, Yukita A, Fukui A, Michiue $T$ et al. Desumoylation activity of Axam, a novel Axin-binding protein, is involved in downregulation of betacatenin. Mol Cell Biol 2002; 22: 3803-3819.

9. Best JL, Ganiatsas S, Agarwal S, Changou A, Salomoni P, Shirihai O et al. SUMO-1 protease-1 regulates gene transcription through PML. Mol Cell 2002; 10: 843-855

10. Zhang H, Saitoh H, Matunis MJ. Enzymes of the SUMO modification pathway localize to filaments of the nuclear pore complex. Mol Cell Biol 2002; 22: 6498-6508.

11. Kim S, Iwao H. Molecular and cellular mechanisms of angiotensin II-mediated cardiovascular and renal diseases. Pharmacol Rev 2000; 52: 11-34.

12. Bailey D, O'Hare P. Characterization of the localization and proteolytic activity of the SUMO-specific protease, SENP1. J Biol Chem 2004; 279: 692-703.

13. Kim YH, Choi $\mathrm{CY}$, Kim Y. Covalent modification of the homeodomain-interacting protein kinase 2 (HIPK2) by the ubiquitin-like protein SUMO-1. Proc Natl Acad Sci USA 1999; 96 : 12350-12355.

14. Ecsedy JA, Michaelson JS, Leder P. Homeodomain-interacting protein kinase 1 modulates Daxx localization, phosphorylation, and transcriptional activity. Mol Cell Biol 2003; 950-960.

15. Kim YH, Choi CY, Lee SJ, Conti MA, Kim Y. Homeodomain-interacting protein kinases, a novel family of co-repressors for homeodomain transcription factors. J Biol Chem 1998; 273: 25875-25879. 
16. Hofmann TG, Moller A, Sirma H, Zentgraf H, Taya Y, Droge W et al. Regulation of p53 activity by its interaction with homeodomain-interacting protein kinase-2. Nat Cell Biol 2002; 4: 1-10.

17. D'Orazi G, Cecchinelli B, Bruno T, Manni I, Higashimoto Y, Saito S et al. Homeodomaininteracting protein kinase-2 phosphorylates p53 at Ser 46 and mediates apoptosis. Nat Cell Biol 2002; 4: 11-19.

18. Zhang Q, Yoshimatsu Y, Hildebrand J, Frisch SM, Goodman RH. Homeodomain interacting protein kinase 2 promotes apoptosis by downregulating the transcriptional corepressor CtBP. Cell 2003; 115: 177-186.

19. Li X, Wang Y, Debatin KM, Hug H. The serine/threonine kinase HIPK2 interacts with TRADD, but not with CD95 or TNF-R1 in 293T cells. Biochem Biophys Res Commun 2000; 277: 513-517.

20. Rochat-Steiner V, Becker K, Micheau O, Schneider P, Burns K, Tschopp J. FIST/HIPK3: a Fas/FADD-interacting serine/threonine kinase that induces FADD phosphorylation and inhibits fas-mediated Jun $\mathrm{NH}(2)$-terminal kinase activation. J Exp Med 2000; 192: $1165-1174$.

21. Li X, Zhang R, Luo D, Park SJ, Wang Q, Kim Y et al. Tumor necrosis factor alpha-induced desumoylation and cytoplasmic translocation of homeodomain-interacting protein kinase 1 are critical for apoptosis signal-regulating kinase 1-JNK/p38 activation. J Biol Chem 2005; 280: 15061-15070.

22. Davis RJ. Signal transduction by the JNK group of MAP kinases. Cell 2000; 103: 239-252.

23. Ichijo H, Nishida E, Irie K, ten DP, Saitoh M, Moriguchi T et al. Induction of apoptosis by ASK1, a mammalian MAPKKK that activates SAPK/JNK and p38 signaling pathways. Science 1997; 275: 90-94

24. Chang HY, Nishitoh $H$, Yang $X$, Ichijo $H$, Baltimore D. Activation of apoptosis signalregulating kinase 1 (ASK1) by the adapter protein Daxx. Science 1998; 281: 1860-1863.

25. Takeda K, Hatai T, Hamazaki TS, Nishitoh H, Saitoh M, Ichijo H. Apoptosis signalregulating kinase 1 (ASK1) induces neuronal differentiation and survival of $\mathrm{PC} 12$ cells. J Biol Chem 2000; 275: 9805-9813.

26. Sayama K, Hanakawa Y, Shirakata Y, Yamasaki K, Sawada Y, Sun L et al. Apoptosis signal-regulating kinase 1 (ASK1) is an intracellular inducer of keratinocyte differentiation. J Biol Chem 2001; 276: 999-1004.

27. Liu Y, Yin G, Surapisitchat J, Berk BC, Min W. Laminar flow inhibits TNF-induced ASK1 activation by preventing dissociation of ASK1 from its inhibitor 14-3-3. J Clin Invest 2001; 107: 917-923

28. Liu Y, Min W. Thioredoxin promotes ASK1 ubiquitination and degradation to inhibit ASK1 mediated apoptosis in a redox activity-independent manner. Cir Res 2002; 90: 1259-1266.

29. Tobiume K, Matsuzawa A, Takahashi T, Nishitoh H, Morita K, Takeda K et al. ASK1 is required for sustained activations of JNK/p38 MAP kinases and apoptosis. EMBO Rep 2001; 2: 222-228.
30. Nishitoh $\mathrm{H}$, Saitoh M, Mochida Y, Takeda $\mathrm{K}$, Nakano $\mathrm{H}$, Rothe $\mathrm{M}$ et al. ASK1 is essential for JNK/SAPK activation by TRAF2. Mol Cell 1998; 2: 389-395.

31. Zhang R, He X, Liu W, Lu M, Hsieh JT, Min W. AIP1 mediates TNF-alpha-induced ASK 1 activation by facilitating dissociation of ASK1 from its inhibitor 14-3-3. J Clin Invest 2003; 111: 1933-1943.

32. Zhang $\mathrm{H}$, Zhang R, Luo Y, D'Alessio A, Pober JS, Min W. AIP1/DAB2IP, a novel member of the Ras-GAP family, transduces TRAF2-induced ASK1-JNK activation. J Biol Chem 2004; 279: 44955-44965.

33. Saitoh M, Nishitoh H, Fujii M, Takeda K, Tobiume K, Sawada $Y$ et al. Mammalian thioredoxin is a direct inhibitor of apoptosis signal-regulating kinase (ASK) 1. EMBO J 1998; 17: 2596-2606.

34. Gotoh Y, Cooper JA. Reactive oxygen species- and dimerization-induced activation of apoptosis signal-regulating kinase 1 in tumor necrosis factor-alpha signal transduction. J Biol Chem 1998; 273: 17477-17482.

35. Zhang L, Chen J, Fu H. Suppression of apoptosis signal-regulating kinase 1-induced cell death by 14-3-3 proteins. Proc Natl Acad Sci USA 1999; 96: 8511-8515.

36. Tobiume K, Saitoh M, Ichijo H. Activation of apoptosis signal-regulating kinase 1 by the stress-induced activating phosphorylation of pre-formed oligomer. J Cell Physiol 2002; 191: 95-104.

37. Kim YH, Sung KS, Lee SJ, Kim YO, Choi CY, Kim Y. Desumoylation of homeodomaininteracting protein kinase 2 (HIPK2) through the cytoplasmic-nuclear shuttling of the SUMO-specific protease SENP1. FEBS Lett 2005; 579: 6272-6278.

38. Min W, Bradley JR, Galbraith JJ, Jones SJ, Ledgerwood EC, Pober JS. The N-termina domains target TNF receptor-associated factor-2 to the nucleus and display transcriptional regulatory activity. J Immunol 1998; 161: 319-324.

39. Zhang R, Luo D, Miao R, Bai L, Ge Q, Sessa WC et al. Hsp90-Akt phosphorylates ASK1 and inhibits ASK1-mediated apoptosis. Oncogene 2005; 24: 3954-3963.

40. Yamaguchi T, Sharma $P$, Athanasiou M, Kumar A, Yamada S, Kuehn MR. Mutation of SENP1/SuPr-2 reveals an essential role for desumoylation in mouse development. $\mathrm{Mol}$ Cell Biol 2005; 25: 5171-5182.

41. Cheng J, Kang X, Zhang S, Yeh ET. SUMO-specific protease 1 is essential for stabilization of HIF1alpha during hypoxia. Cell 2007; 131: 584-595.

42. Charette SJ, Lambert $\mathrm{H}$, Landry J. A kinase-independent function of Ask1 in caspaseindependent cell death. J Biol Chem 2001; 276: 36071-36074.

43. Elbashir SM, Harborth J, Lendeckel W, Yalcin A, Weber K, Tuschl T. Duplexes of 21. nucleotide RNAs mediate RNA interference in cultured mammalian cells. Nature 2001; 411: 494-498.

44. He Y, Zhang W, Zhang R, Zhang H, Min W. SOCS1 inhibits tumor necrosis factor-induced activation of ASK1-JNK inflammatory signaling by mediating ASK1 degradation. J Biol Chem 2006; 281: 5559-5566. 